\title{
Planar substrate-binding site dictates the specificity of ECF-type nickel/cobalt transporters
}

You $\mathrm{Yu}^{1,2}$, Mingze Zhou ${ }^{1,2}$, Franziska Kirsch ${ }^{3}$, Congqiao Xu ${ }^{4}$, Li Zhang ${ }^{1,2}$, Yu Wang ${ }^{5}$, Zheng Jiang ${ }^{5}$, Na Wang ${ }^{1,2}$, Jun $\mathrm{Li}^{4}$, Thomas Eitinger ${ }^{3}$, Maojun Yang ${ }^{1,2}$

${ }^{I}$ MOE Key Laboratory of Protein Sciences, Tsinghua-Peking Center for Life Sciences, School of Life Sciences, Tsinghua University, Beijing 100084, China; '2Department of Pharmacology and Pharmaceutical Sciences, School of Medicine, Tsinghua University, Beijing 100084, China; ${ }^{3}$ Humboldt-Universität zu Berlin, Institut für Biologie/Mikrobiologie, 10115 Berlin, Germany; ${ }^{4}$ Department of Chemistry, Tsinghua University, Beijing 100084, China; ${ }^{5}$ Shanghai Synchrotron Radiation Facilities, Shanghai Institute of Applied Physics, Chinese Academy of Sciences, Shanghai 201204, China

The energy-coupling factor (ECF) transporters are multi-subunit protein complexes that mediate uptake of transition-metal ions and vitamins in about $50 \%$ of the prokaryotes, including bacteria and archaea. Biological and structural studies have been focused on ECF transporters for vitamins, but the molecular mechanism by which ECF systems transport metal ions from the environment remains unknown. Here we report the first crystal structure of a NikM, TtNikM2, the substrate-binding component (S component) of an ECF-type nickel transporter from Thermoanaerobacter tengcongensis. In contrast to the structures of the vitamin-specific $\mathrm{S}$ proteins with six transmembrane segments (TSs), $T t$ NikM2 possesses an additional TS at its N-terminal region, resulting in an extracellular N-terminus. The highly conserved N-terminal loop inserts into the center of $T t$ NikM2 and occludes a region corresponding to the substrate-binding sites of the vitamin-specific $S$ components. Nickel binds to NikM via its coordination to four nitrogen atoms, which are derived from Met1, His2 and His67 residues. These nitrogen atoms form an approximately square-planar geometry, similar to that of the metal ion-binding sites in the amino-terminal $\mathrm{Cu}^{2+}-\mathrm{and}^{2 i^{2+}}$-binding (ATCUN) motif. Replacements of residues in NikM contributing to nickel coordination compromised the Ni-transport activity. Furthermore, systematic quantum chemical investigation indicated that this geometry enables NikM to also selectively recognize $\mathrm{Co}^{2+}$. Indeed, the structure of $T t \mathrm{NikM} 2$ containing a bound $\mathrm{Co}^{2+}$ ion has almost no conformational change compared to the structure that contains a nickel ion. Together, our data reveal an evolutionarily conserved mechanism underlying the metal selectivity of EcfS proteins, and provide insights into the ion-translocation process mediated by ECF transporters.

Keywords: energy-coupling factor transporter; nickel and cobalt transporters; crystal structure

Cell Research (2014) 24:267-277. doi:10.1038/cr.2013.172; published online 24 December 2013

\section{Introduction}

The energy-coupling factor (ECF) transporters are

Correspondence: Maojun Yang ${ }^{\mathrm{a}}$, Thomas Eitinger ${ }^{\mathrm{b}}, \mathrm{Jun}_{\mathrm{Li}}^{\mathrm{c}}$

${ }^{a}$ E-mail: maojunyang@tsinghua.edu.cn

Tel: +86-10-6278-9400; Fax: +86-10-6279-2736

${ }^{b}$ E-mail: thomas.eitinger@cms.hu-berlin.de

Tel: +49-30-2093-8103; Fax: +49-30-2093-8102

${ }^{c}$ E-mail: junli@tsinghua.edu.cn

Tel: +86-10-6279-5381; Fax: +86-10-6279-7472

Received 27 August 2013; revised 14 October 2013; accepted 29 October 2013; published online 24 December 2013 multi-subunit protein complexes that mediate uptake of transition-metal ions and vitamins across the membrane in many bacteria and archaea [1-7]. They consist of a substrate-specific integral membrane protein (S component, EcfS), a moderately conserved but ubiquitous transmembrane protein ( $\mathrm{T}$ component, EcfT), and homo- or heterooligomeric ABC ATPases (A components, EcfA). ECF transporters have been classified into two types. For type-1 transporters, all the components are encoded in the same operon, whereas for type- 2 transporters, the EcfAA'T module is encoded in an operon, but the genes for $\mathrm{S}$ units are scattered around the genome. The S com- 
ponents interact with the EcfAA'T module to form a holotransporter complex. Previous structural studies have been focused on isolated S components, such as the riboflavin- [5], thiamine- [8], and biotin-specific [1] S units (RibU, ThiT, BioY), and on two type-2 holotransporters containing a folate-specific S component (FolT) [3] and an $\mathrm{S}$ component with predicted specificity for pyridoxine (PdxU, named HmpT in the original work [4]), respectively. All of these EcfS proteins have a similar structural fold with six transmembrane helices. The isolated S components were crystallized with their substrates that bind to a cavity facing the outside of the membrane. This observation correlates well with previous analyses of binding affinity that identified extremely low dissociation constants in the (sub)nanomolar range.

ECF metal transporters belong to type-1 transporters. The mechanism by which ECF metal transporters selectively recognize metal ions remains largely unknown. As a unique feature of ECF metal transporters, function of the $\mathrm{S}$ component (NikM and CbiM for nickel and cobalt transporters, respectively) relies on additional small transmembrane proteins represented by the " $\mathrm{N}$ " proteins in most cases or "K plus L" proteins for the rest. ECF-type nickel and cobalt transporters are the most common uptake systems for these metals in prokaryotes [6, 9-11]. The vital importance of NikMNQO and CbiMNQO transporters (Q and $\mathrm{O}$ represent the $\mathrm{T}$ and $\mathrm{A}$ components, respectively) in the biology of microorganisms stems from the indispensable roles of these two transition-metal ions in cells.

Nickel is a cofactor of a variety of metalloenzymes involved in energy and nitrogen metabolism, carbon dioxide fixation and detoxification processes [9, 12-14]. Cobalt is a key constituent of vitamin $B_{12}$ [15]. Vitamin $\mathrm{B}_{12}$ is the largest and the most structurally complicated water-soluble vitamin, and contains the biochemically rare element cobalt. In contrast to many archaea and bacteria that have the enzymes required for vitamin $B_{12}$ synthesis, fungi, higher plants and animals are unable to synthesize vitamin $\mathrm{B}_{12}$. Therefore, microorganisms are the only sources of vitamin $B_{12}$ in nature [16]. Vitamin $\mathrm{B}_{12}$ is involved in many critical biological processes in cells, such as DNA synthesis, fatty acid degradation and energy conservation $[17,18]$. Vitamin $B_{12}$ deficiency can potentially cause severe and irreversible damage, especially to the brain and nervous system, and can also cause symptoms of mania and psychosis [19]. Although the biochemical mechanism for vitamin $\mathrm{B}_{12}$ synthesis has been vastly studied $[20,21]$, the molecular mechanism by which the cells take up cobalt from the medium remains unclear. Several types of primary and secondary active nickel and cobalt ion transporters have been reported [12], however in no case has the mechanism of selective metal binding and transport been elucidated.

In the present study, we solved the crystal structure of $T t$ NikM2, the S component of an ECF-type nickel transporter from Thermoanaerobacter tengcongensis. The overall structure of $T t \mathrm{NikM} 2$ resembles the fold of the previously characterized S components of type- $2 \mathrm{ECF}$ transporters. It differs from them, however, by possessing an additional, $\mathrm{N}$-terminal transmembrane segment that positions the $\mathrm{N}$-terminus to the extracellular side of the membrane. The highly conserved N-terminal loop is crucial for the selection of metal ions. It inserts into a region corresponding to the substrate-binding sites of the vitamin-specific $\mathrm{S}$ components and contributes significantly to metal ion binding by donating three nitrogen atoms. In total, four nitrogen atoms (the free amino group of Met1, the backbone nitrogen and the imidazole nitrogen of His2, and the imidazole nitrogen of His67) form an approximately square-planar geometry for the specific recognition of $\mathrm{Ni}^{2+}$. Structural and quantum chemical analyses indicate that this architecture is also suitable for binding $\mathrm{Co}^{2+}$, which is consistent with functional genomics analyses showing that the key residues for ion recognition are highly conserved among NikM/CbiM proteins from different species. Replacement of residues associated with metal ion coordination reduces the $\mathrm{Ni}^{2+}$-uptake activity. Our structural studies, along with functional and theoretical analyses, reveal an evolutionarily conserved mechanism underlying the ion selectivity of this important class of transporters.

\section{Results and Discussion}

\section{TtNikM2 is a nickel-specific S component}

Through functional genomics analyses, we identified two putative nickel-specific $\mathrm{S}$ components in T. tengcongensis: TTE0249 (TtNikM) and TTE1695 (Tt NikM2) (Supplementary information, Figures S1-S3 and Table $\mathrm{S} 1)$. Indeed, the X-ray fluorescence spectrum and the anomalous scattering signal screening results demonstrate that the metal ion present in the $T t \mathrm{NikM} 2$ crystals is a nickel rather than a cobalt or iron ion (Figure 1A, Supplementary information, Figure S4 and Table S2). Using the nickel ion anomalous signal, we solved the structure of the $T t \mathrm{NikM} 2$ protein with a C-terminal truncation (amino acids 1 to 209) at $1.83 \AA$ resolution (Supplementary information, Figures S4-S6). The full-length $T t$ NikM2 (amino acids 1 to 230) structure was solved by molecular replacement method at $3.2 \AA$ resolution (Figure $1 B$ and Supplementary information, Table S3). The rmsd between the two structures is $0.25 \AA$ with 209 aligned $\mathrm{C} \alpha$ atoms. In the crystal, each asymmetric unit contains a single peptide and one nickel ion. The linker between the 
A

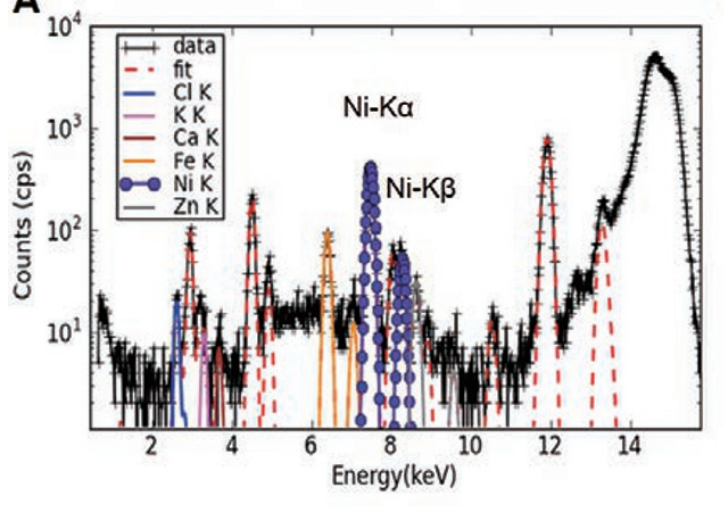

B

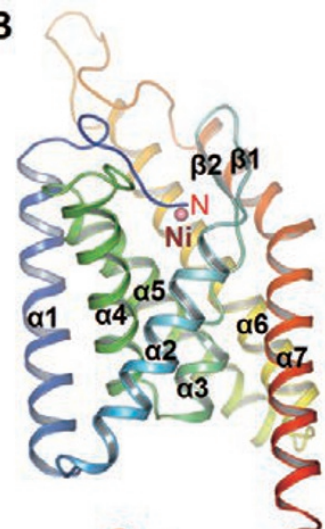

C
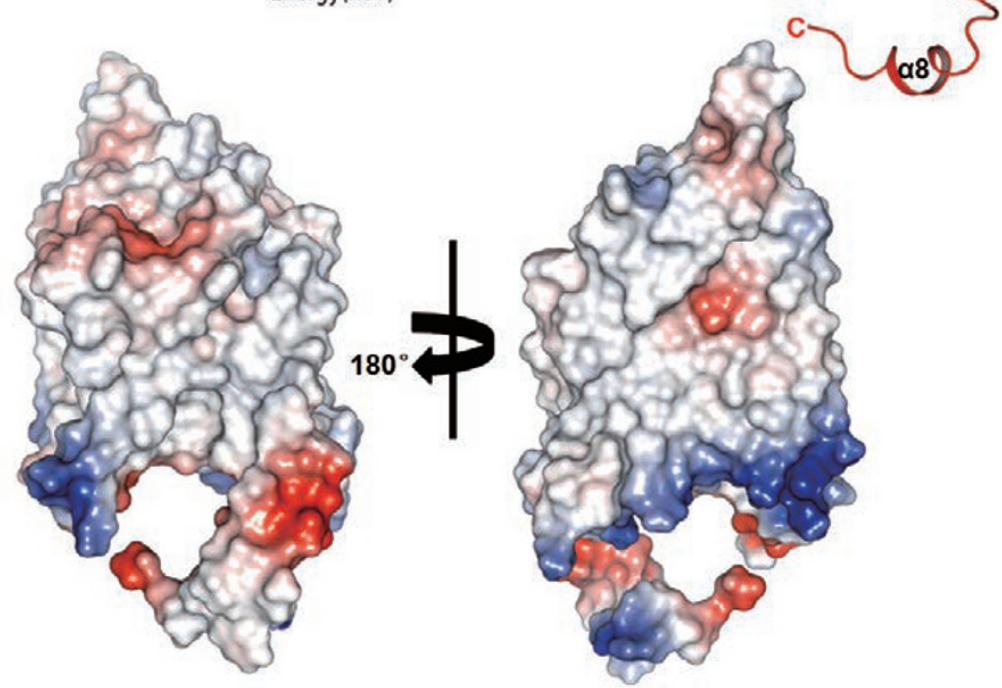

Figure 1 Overall structure of TtNikM2. (A) The X-ray fluorescence spectrum as fitted shows multiple elements, e.g., Cl, $\mathrm{K}$, $\mathrm{Ca}, \mathrm{Fe}, \mathrm{Ni}$ and $\mathrm{Zn}$ ions. The nickel ion's $\mathrm{K} \alpha$ and $\mathrm{K} \beta$ emission lines are shown as solid-dot purple lines. (B) Overall structure of TtNikM2. The TtNikM2 is shown in cartoon model, colored by a rainbow fashion. The $\mathrm{Ni}^{2+}$ ion is shown as a sphere. The secondary structural elements, $\mathrm{N}$ - and C-termini are indicated. (C) TtNikM2 is represented as an electrostatic surface model and two views related by $180^{\circ}$ rotation along the vertical axis are shown. The blue, white, and red shadings represent positively charged, neutral, and negatively charged surface regions, respectively.

$\alpha 2$ and $\alpha 3$ helices comprises two anti-parallel $\beta$-strands (Figure 1B). The C-terminal end of $T t \mathrm{NikM} 2$ forms a short $\alpha 8$ helix (amino acids 216-end). The surface of this $\alpha 8$ helix towards the cytoplasm is mainly hydrophobic, which suggests that this helix might interact with other components in the cytoplasm (Supplementary information, Figure S7). The overall structure of $T t \mathrm{NikM} 2$ is hydrophobic with a positively charged belt facing the cytoplasmic side of the membrane, which is in agreement with the positive-inside rule $[22,23]$ (Figure 1C).

\section{TtNikM2 has a unique N-terminus}

The overall structure of $T t \mathrm{NikM} 2$ comprises seven transmembrane segments with a short helix at the C-terminus (Figures 1B and 2), and the additional transmembrane segment at the N-terminus distinguishes it from the substrate- binding components of other ECF-type transporters [1-5, 8]. The transmembrane segments 2-7 resemble the folds of RibU, ThiT and BioY, with rmsds of $3.27 \AA, 2.59 \AA$, and $2.88 \AA$ for 150,133 , and 140 aligned $\mathrm{C} \alpha$ atoms between $T t \mathrm{NikM} 2$ and the $\mathrm{S}$ units, respectively (Figure 2, left panel). The N-terminal loop (amino acids 1-9) in front of the $\alpha 1$ helix (amino acids 10-34) inserts into the center of $T t \mathrm{NikM} 2$ and occludes a region corresponding to the substrate-binding sites of the vitamin-specific $\mathrm{S}$ components, ruling out the possibility that $T t \mathrm{NikM} 2$ recognizes any vitamins (Figure 2 , right panel).

The unique N-terminus of NikM is required for substrate binding

Previous studies have suggested that the amino group and the length of the N-terminal stretch of NikM/CbiM 
proteins are essential determinants in metal recognition and transport [24]. The structure of $T t \mathrm{NikM} 2$ reveals that the highly conserved first 9-residue loop inserts into the center of $T t \mathrm{NikM} 2$ and forms a series of hydrogen bonds with the surrounding residues (Figure 3B and Supplementary information, Figure S2). Interestingly, the free $\mathrm{N}$-terminal amino group of Met1 directly coordinates the nickel ion, and its side chain is stabilized by a deep, hydrophobic pocket that is mainly formed by Met113, Ala150, Ala151 and His190 residues (Figure 3B). The main chain amide nitrogen and the imidazole nitrogen of His 2 also contribute to nickel binding. These three nitrogen atoms together with the side chain imidazole nitrogen of His67 form the nickel-binding site. This observation is consistent with previous studies showing that adding an additional Met at the N-terminus or inserting an Ala between Met1 and His2 resulted in the loss of transport activity [24]. Furthermore, the first 9 residues, and Gln94, Gly101, Gly102 and Asn109 that interact with the N-terminal loop, are highly conserved in NikM/ CbiM proteins from different bacteria species (Figure 3B, 3C and Supplementary information, Figure S2), suggesting a similar metal-recognizing mechanism among these proteins.

Four nitrogen atoms form an approximately squareplanar substrate-binding site

The nickel ion is chelated by the nitrogen atom of the

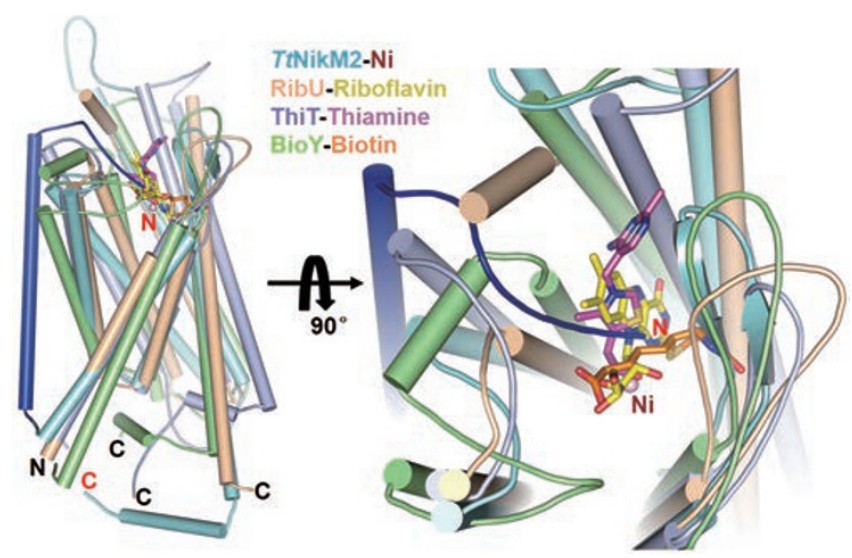

Figure 2 TtNikM2 has an additional transmembrane helix and an extra N-terminal loop compared to vitamin-specific $S$ components. For TtNikM2, the extra N-terminal helix and loop are shown in blue and the rest of the protein is colored in cyan. RibU, ThiT, BioY are colored in wheat, slate, and lime, respectively. The right panel shows substrate-binding sites with $90^{\circ}$ rotation to the left panel. The rioflavin, thiamine and biotin are colored in yellow, magenta and orange in sticks, respectively. The $\mathrm{Ni}^{2+}$ is shown as a lightpink sphere. free N-terminal amino group of Met1, the main chain amide nitrogen and the $\mathrm{N} \delta 1$ atom of His2, and the $\mathrm{N} \varepsilon 2$ atom of His67 from the $\alpha 2-\alpha 3$ linker (Figure 4A). Unlike in other divalent metal transporters $[11,25,26]$ and in nickel-binding proteins $[12,14]$, the four nitrogen atoms form an approximately square-planar geometry with slightly different distances from the nickel to the nitrogen atoms (Figure 4A and Supplementary information, Figure S8A). This arrangement is reminiscent of the approximately square-planar geometry of the aminoterminal $\mathrm{Cu}^{2+}$ - and $\mathrm{Ni}^{2+}$-binding (ATCUN) motif, which has been found in certain species of albumins (e.g., HSA, BSA, and RSA), human sperm protamine P2a, neuromedins $\mathrm{C}$ and $\mathrm{K}$, and histatins [27-35]. Unlike the metal ion binding mode in $T t \mathrm{NikM} 2$, the ATCUN motif consists of the N-terminal amine, the backbone amide nitrogens of the second and third residues, and an imidazole nitrogen of His4 (Supplementary information, Figure S8). Consistent with our structural observation, the nickel $K$-edge Xray absorption near-edge structure (XANES) results also indicate a square-planar geometry around the nickel ion of $T t \mathrm{NikM} 2$ in solution. The shoulder feature at 8339.5 $\mathrm{eV}$ present in $T t \mathrm{NikM} 2$ and in $\mathrm{Ni}(\mathrm{dmg})_{2}$ is attributed to the $1 \mathrm{~s}-$ to $-4 \mathrm{p}_{\mathrm{z}}$ transition [36], indicative of a characteristic of the almost square-planar coordination geometry around the nickel (Figure 4B).

The nickel-chelating residues are stabilized by a series of hydrogen bonds (Figure 4A). The hydrogen atoms from the $\mathrm{N}$-terminal free amine form hydrogen bonds with the carbonyl group of Thr65 and a water molecule (Figure 4C). This water molecule forms additional hydrogen bonds with the carbonyl group of His67 and the $\mathrm{N} \varepsilon 2$ atom of His 190. The N $\delta 1$ atom of His190 forms a hydrogen bond with the carbonyl group of Met186. The $\mathrm{N} \varepsilon 2$ atom of His2 forms a hydrogen bond with the side chain of Gln94. The N $\delta 1$ atom of His67 forms a hydrogen bond with the carbonyl group of Ala68, and the side chain of Glu198 forms another hydrogen bond with the backbone amide of Ile69 (Figure 4C). Interestingly, the $\mathrm{Ni}^{2+}$ has two positive charges, but from the structural analyses we predict that only one hydrogen from the main-chain amide of His 2 could be deprotonated. We propose that this elaborate hydrogen bond network not only stabilizes the planar nickel ion-binding geometry, but also contributes to the charge neutralization. More interestingly, these residues involved in the formation of the hydrogen bond network are highly conserved among the NikM/CbiM proteins from different species (Supplementary information, Figure S2), suggesting that the mechanism of nickel or cobalt recognition is most likely conserved.

To confirm the biological relevance of the structural observations, we attempted to examine $\mathrm{Ni}^{2+}$ uptake in 

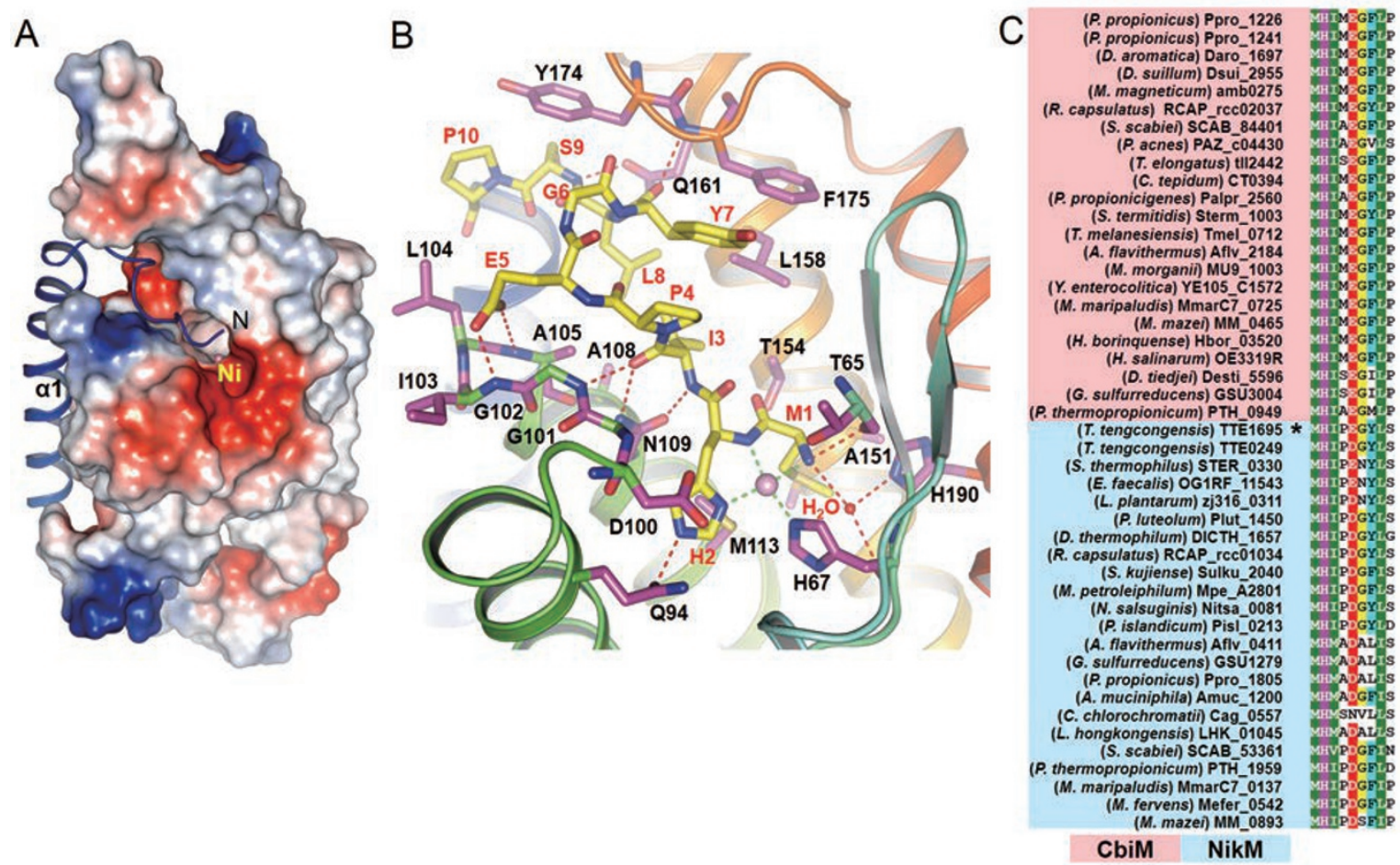

Figure 3 The $\mathrm{N}$-terminus of $T t \mathrm{NikM} 2$ is responsible for $\mathrm{Ni}^{2+}$ binding. (A) The N-terminal loop of $T t \mathrm{NikM} 2$ inserts into a deep pocket. The $\alpha 1$-helix and the $\mathrm{N}$-terminal loop are shown in ribbon representation and the rest of the protein is shown in an electrostatic potential surface. The blue, white, and red shadings represent positively charged, neutral, and negatively charged surface regions, respectively. The $\mathrm{Ni}^{2+}$ ion is shown as a sphere. (B) A close view of the $\mathrm{N}$-terminal substrate-binding pocket. The residues from $\mathrm{N}$-terminal loop and from the rest of the protein are shown in yellow and magenta sticks, respectively. The water molecule is shown as a red sphere. The $\mathrm{Ni}^{2+}$-nitrogen coordinate bonds and hydrogen bonds are shown as green and red dashed lines, respectively. (C) The sequence alignment of the highly conserved N-terminus (amino acids 1-9) of CbiMs and NikMs from different species.

recombinant Eischerichia coli cells producing TtNikM2N2. It was previously shown that M plus N proteins (in the absence of $\mathrm{Q}$ and $\mathrm{O}$ components) are the minimal unit of ECF-type metal transporters required to mediate basal levels of substrate uptake $[6,24]$. However, we failed to detect any metal ion uptake above background. It is possible that $T t \mathrm{NikM} 2$ and $T t \mathrm{NikN} 2$ from the thermophilic $T$. tengcongensis do not assemble into a functional transporter or it is likely that other components are required for the establishment of a functional transporter in the mesophilic E. coli host. Moreover, M and $\mathrm{N}$ proteins were previously shown to only loosely interact with each other [24]. Therefore, we used the homologous nickel transporter, $R c \mathrm{Nik}(\mathrm{MN}) \mathrm{QO}$, from the purple bacterium Rhodobacter capsulatus to test the biological relevance of the structural observations (Figure 4D). In this system, the NikM and NikN proteins are fused naturally to form a single $\mathrm{Nik}(\mathrm{MN})$ peptide. Given the sequence similarity between the nickel transporters from $T$. tengcongensis and $R$. capsulatus (Supplemen- tary information, Figure S9), we replaced the structurally important residues in the NikM part of $R c \operatorname{Nik}(\mathrm{MN})$ QO and tested the effects on nickel-uptake activity. Replacement of His67 (RcNik( $\left.\left.\mathrm{M}^{\mathrm{His67 \textrm {Ala }}} \mathrm{N}\right) \mathrm{QO}\right)$, which is involved in nickel binding, abolished transport activity. Likewise, replacements of residues stabilizing the planar geometry greatly (His194Ala, His194Glu, Glu202Gln, Glu202Asn, Glu202A) or at least significantly (Thr65Ala) impaired the $\mathrm{Ni}^{2+}$-uptake activity, whereas replacements that preserve hydrogen bonds (Gln94Glu, His194Gln, Glu202Asp) exhibited only a moderate effect (Figure 4D). The latter finding is consistent with our prediction that the hydrogen bond network contributes to the charge neutralization (Figure 4D). The essential roles of Met1 and His 2 for the $\mathrm{Ni}^{2+}$-uptake activity have been reported $[6,24]$.

\section{The substrate-binding site dictates the ion specificity}

Structural analysis suggests that only the hydrogen atom from the backbone amide of His 2 can be deproton- 

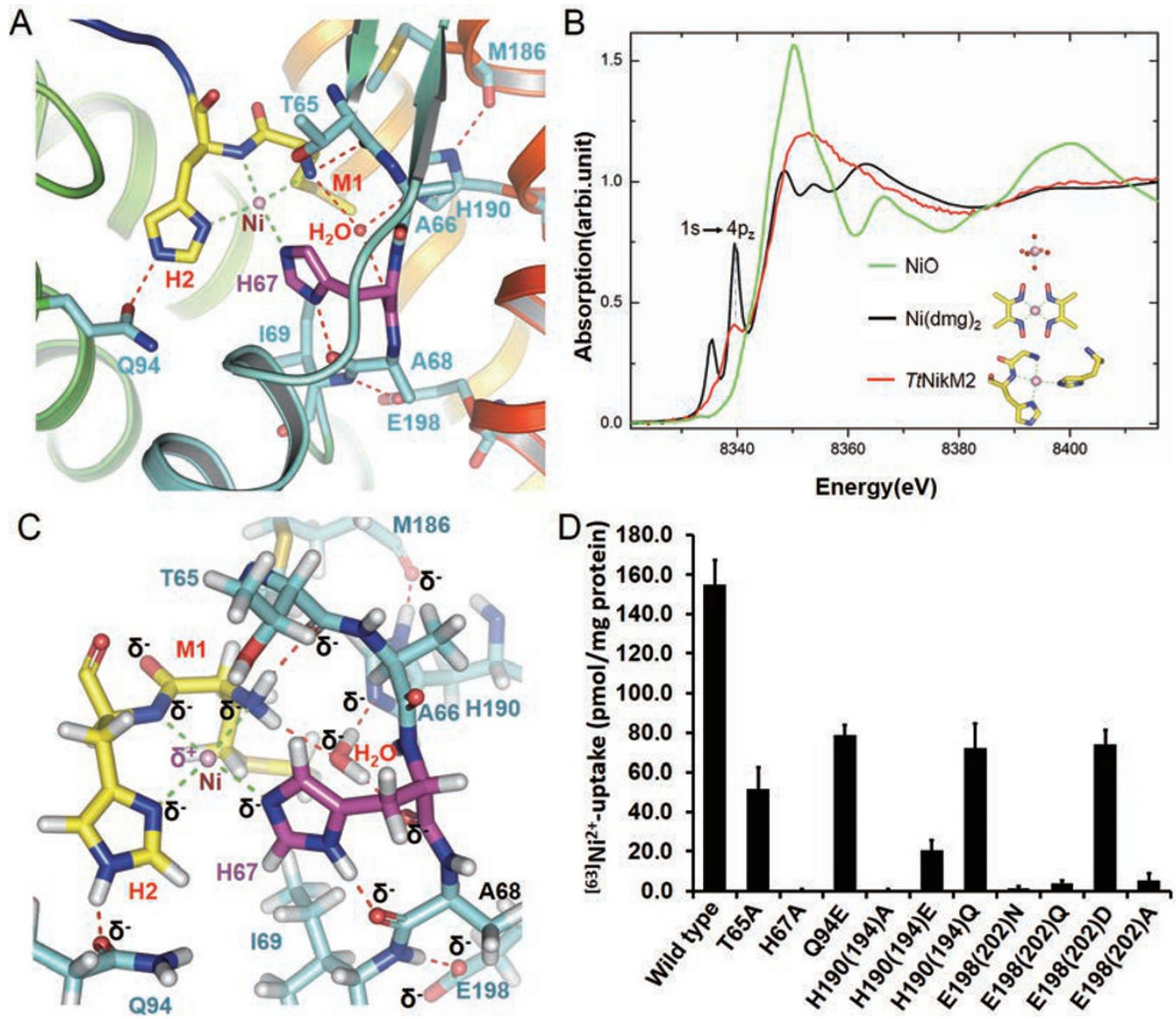

Figure 4 The approximately square-planar geometry of $\mathrm{Ni}^{2+}$-binding site. (A) The detailed binding mode of the $\mathrm{Ni}^{2+}$. The residues involved in $\mathrm{Ni}^{2+}$ coordination and stabilization of the binding pocket are shown as sticks. The $\mathrm{Ni}^{2+}$-nitrogen coordinate bonds and hydrogen bonds are shown as green and red dashed lines, respectively. (B) Comparison of the Ni K-edge XANES spectra of the $\mathrm{NiO}, \mathrm{Ni}(\mathrm{dmg})_{2}$ in solid and TtNikM2 in solution. The observed distinct shoulder feature at $8339.5 \mathrm{eV}$ present in the $T t \mathrm{NikM} 2$ and $\mathrm{Ni}(\mathrm{dmg})_{2}$ is attributed to the $1 \mathrm{~s}$-to- $4 \mathrm{p}_{\mathrm{z}}$ electronic transition in a square-planar metal complex. The structures of the $\mathrm{Ni}^{2+}$-binding sites of $\mathrm{NiO}, \mathrm{Ni}(\mathrm{dmg})_{2}$ and $T t \mathrm{NikM} 2$ are shown in sticks. (C) The electron distribution of the $\mathrm{Ni}^{2+}-$ binding pocket. The residues are shown in stick model, with hydrogen atoms displayed. The distributions of electrons are indicated by $\delta^{-}$and $\delta^{+}$. (D) $\mathrm{Ni}^{2+}$-uptake activity of $E$. coli cells containing the RcNik(MN)QO transporter from R. capsulatus with wild-type or mutant $R c N i k M$. Each assay was performed in triplicate. Mean values \pm standard deviations are shown. Numbers in parentheses correspond to residue positions in RcNikM, where the numbering differs between RcNikM and TtNikM.

ated upon coordination of $\mathrm{Ni}^{2+}$ in the cavity formed by four $\mathrm{N}$-atoms (Figure $5 \mathrm{~A}$ ). $\mathrm{Ni}^{2+}$ has two positive charges while the deprotonated cavity possesses one negative charge. It is thus imperative to understand how the protein neutralizes the extra positive charge and how this metal-binding geometry dictates ion selectivity. To elucidate the nature of the selectivity of the $T t \mathrm{NikM} 2$ protein for divalent nickel ion, we have performed systematic quantum chemical investigations of the chemical bonding, charge distribution and binding strength of the protein with a series of biologically relevant divalent metal cations $\left(\mathrm{M}=\mathrm{Ca}^{2+}, \mathrm{Mg}^{2+}, \mathrm{Fe}^{2+}, \mathrm{Co}^{2+}, \mathrm{Ni}^{2+}, \mathrm{Cu}^{2+}\right.$ and $\left.\mathrm{Zn}^{2+}\right)$ (Figure 5B and 5C). We selected a fragment $\mathrm{L}^{-}$(Figure
$5 \mathrm{~A}$ ) of the local structure of the protein and the dangling bonds are saturated with hydrogen atoms to fulfill the correct valence states of each atom. As the protein is deprotonated upon coordination of the metal ions, the binding ligand is negatively charged. The Gibbs free energies for $\mathrm{M}^{2+}+\mathrm{L}^{-} \rightarrow \mathrm{ML}^{+}$were calculated as $\Delta \mathrm{G}=\mathrm{G}\left(\mathrm{ML}^{+}\right)$$\mathrm{G}\left(\mathrm{M}^{2+}\right)-\mathrm{G}\left(\mathrm{L}^{-}\right)$, where the entropy effects are included along with the quantum mechanical-binding energies. The negative energy indicates the binding is thermodynamically exothermic. The metal-binding constants $K_{\mathrm{p}}$ were calculated from $\Delta \mathrm{G}=-\mathrm{RT} \ln K_{\mathrm{p}}$. The energies of the $\mathrm{M}^{2+}$ and $\mathrm{ML}^{+}$ions are determined with all possible multiplets and spin-multiplicities. The lowest-energy electron 

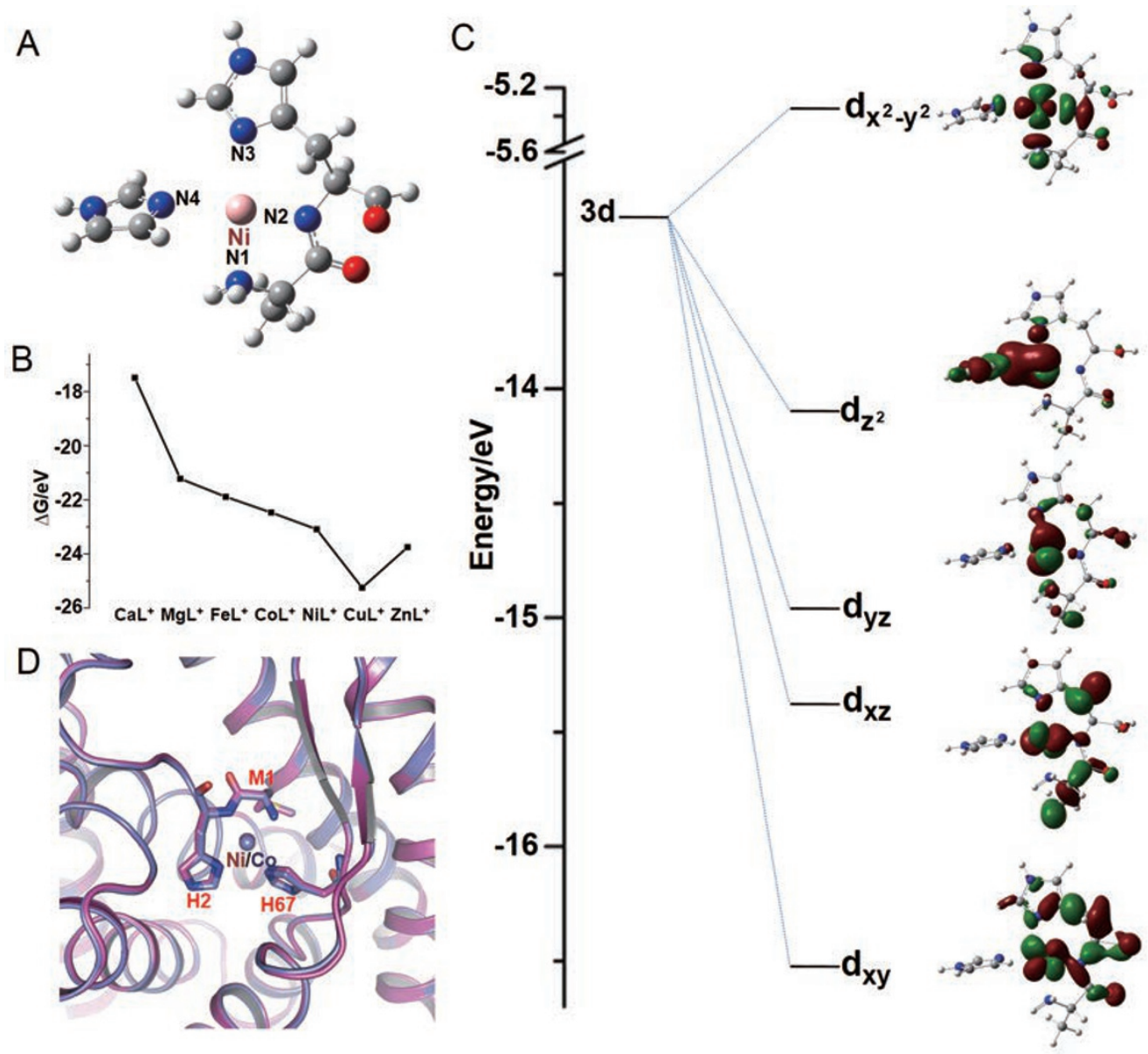

Figure 5 The approximately square-planar geometry of the metal-binding site dictates substrate specificity of $T t N i k M 2$. (A) Structure of the TtNikM2 nickel coordinate cavity. The nitrogen atoms are defined as N1, N2, N3, and N4. (B) Binding energy of $\mathrm{M}^{2+}+\mathrm{L}^{-} \rightarrow \mathrm{ML}^{+}$(M represents different divalent metal ions) as calculated with $\mathrm{BHaH}$ functional. (C) Splitting of the $3 \mathrm{~d}$ orbital in $\mathrm{NiL}^{+}$and $3 \mathrm{D}$ contours (isovalue $=0.03$ ) of Kohn-Sham molecular orbitals. The calculated Kohn-Sham energy levels and the contour diagrams of the frontier molecular orbitals of $\mathrm{NiL}^{+}$are shown, where $\mathrm{Ni}^{2+}$ has a $\mathrm{d}^{8}$ electron configuration. (D) Structural alignment of the TtNikM2-nickel and TtNikM2-cobalt structures. The structures with nickel and cobalt are shown in magenta and slate, respectively. The residues binding the metal ions are shown in sticks.

configurations are used as the references for the Gibbs free energies. The atomic electron configurations of $\mathrm{M}^{2+}$ used are consistent with those proposed by Johnson et al. [37].

The density functional theory (DFT) calculation [3739] results indicate that when the divalent metal cations bind to the substrate-binding cavity, $\mathrm{Ca}^{2+}$ and $\mathrm{Mg}^{2+}$ have the least binding energy due to the pure ionic interaction (Figure 5B, and Supplementary information, Table S4), while the transition-metal ions have larger binding energies due to both ionic and covalent interactions between the $\mathrm{N}$-atoms and the metal 3d-orbitals (see the contours of the $\mathrm{NiL}^{+}$orbitals in Figure 5C). In the structures, ligand field theory (LFT) predicts that the d-orbital manifold of the metal ions will split into five groups, with one strong anti-bonding orbital $\left(\mathrm{d}_{\mathrm{x} 2-\mathrm{y}_{2}}\right)$ lying above the other four d-orbitals (Figure 5C). This ligand field splitting pattern indicates that a $\mathrm{d}^{8}$ configuration should have larger binding energy than the others. Indeed, the DFT calculations support this qualitative conclusion, with $\mathrm{Co}^{2+}$ $\left(\mathrm{d}^{7}\right)$ and $\mathrm{Ni}^{2+}\left(\mathrm{d}^{8}\right)$ having Gibbs free energies of -22.47 $\mathrm{eV}$ and $-23.09 \mathrm{eV}$ (electron volt), respectively (Figure $5 \mathrm{~B}$ and Supplementary information, Table S4). The $\mathrm{Cu}^{2+}$ $\left(d^{9}\right)$ ion tends to have even larger free energy, but this ion might prefer a different local structure due to the JahnTeller effect.

The calculated atomic polar tensor (APT)-derived charges [40] indicate that the four $\mathrm{N}$-atoms have different charges (Supplementary information, Table S5). Their charges are not only consistent with the distance 
difference between the Ni- $\mathrm{N}_{m}(\mathrm{~m}=1,2,3,4)$ coordination bonds (Figure 5A and Supplementary information, Figure S8A), but also consistent with the notion that the surrounding hydrogen-bond network contributes to the charge neutralization (Figure 4C). These data suggest that the metal ion type and the metal-ligand bonding strength might constitute the most important elements to determine the metal ion selectivity. To confirm the theoretical results, we purified and co-crystallized $T t \mathrm{NikM} 2_{1-209}$ with $\mathrm{CoCl}_{2}$ and solved the structure at $2.5 \AA$ resolution (Supplementary information, Figure S10 and Table S3). The structure has a $0.22 \AA$ rmsd with the structure that contains nickel ion and has almost no conformational changes (Figure 5D), which suggests that $T t \mathrm{NikM} 2$ could bind both nickel and/or cobalt ions. These results confirm the hypothesis that $\mathrm{CbiM}$ and NikM proteins utilize similar mechanisms to recognize their substrates. They are also consistent with the previous biological observation that the CbiMNQO complexes of both Salmonella enterica serovar Typhimurium and $R$. capsulatus not only import cobalt ions but also transport nickel ions [6].

\section{Conclusions}

ECF transporters have evolved into two types with high selectivity for either vitamins or transition-metal ions. Our studies unravel that the specific $\mathrm{Co}^{2+}$ and/or $\mathrm{Ni}^{2+}$ recognition by NikM is achieved by the presence of an additional N-terminal transmembrane helix that enables the very $\mathrm{N}$-terminal end of the protein with its three metal ligands to deeply insert into the substrate-binding site, thereby preventing entrance of larger molecules and establishing ion selectivity. This represents another fascinating feature of the structurally and mechanistically unusual ECF transporters. The mechanistic studies of how these protein complexes exert their micronutrient-transport functions could be valuable for the development of new antibiotics since ECF transporters are essential for pathogenic bacteria.

\section{Materials and Methods}

Genome-wide analysis of phylogenetic relationships of Nickel and Cobalt transporters

The $c b i M$ and $n i k M$ genes were obtained from KEGG (the Kyoto Encyclopedia of Genes and Genomes) ORTHOLOGY Database (KEGG ORTHOLOGY Entry K02007) (Supplementary information, Table S1). The collected sequences were aligned using the MUSCLE (multiple sequence comparison by log-expectation) program [41] and further adjusted manually using BIOEDIT. The optimal substitution model of amino acid substitution $(\mathrm{RtREV}+\mathrm{G}+\mathrm{F})$ was selected using the modelGenerator version 0.84 program [42]. The phylogenetic tree in Supplementary information, Figure S1 was constructed using the maximum likelihood method with PhyML software [43]. One hundred bootstrap replicates were per- formed to obtain the confidence support.

\section{The X-ray fluorescence spectrum assay}

The microscopic X-ray fluorescence ( $\mu$-XRF) measurements are performed at Beijing Synchrotron Radiation Facility (BSRF) 4W1B endstation, which runs $2.5 \mathrm{GeV}$ with current from 150 $\mathrm{mA}$ to $250 \mathrm{~mA}$. The X-ray beam impinged on $T t \mathrm{NikM} 2$ crystal is monochromatized at $15 \mathrm{keV}$ and is focused down to $50 \mu \mathrm{m}$ in diameter by the poly-capillary lens. The detection limit of the experimental setup is $\sim \mu \mathrm{g} / \mathrm{g}$. The $T t \mathrm{NikM} 2$ crystal is pasted on XRF tape (TF-500) at room temperature and then attached to a frameshape sample holder. The $\mathrm{Si}(\mathrm{Li})$ solid state detector is used to detect X-ray fluorescence emission lines with live time of $100 \mathrm{~s}$ per point. Several XRF spectra were collected at the sample and the blank tape, respectively. The data reduction and process were performed using PyMCA package with linear polynomial background accounting for scattering [44]. Because the X-ray might penetrate the sample and strike the tape, the XRF spectrum of pure $T t \mathrm{NikM} 2$ crystal is achieved by subtracting the XRF spectrum of blank tape.

\section{Protein preparation}

PCR-amplified full-length nikM2 from $T$. tengcongensis was subcloned into $\mathrm{pET} 21 \mathrm{~b}$ vector with a TEV protease site before the His-tag. Overexpression of TtnikM2 was induced in E. coli BL21 (DE3) by $0.5 \mathrm{mM}$ isopropyl- $\beta$-D-thiogalactoside (IPTG) when the cell density reached $\mathrm{OD}_{600}=1.0$. After growth in Terrific Broth for $3 \mathrm{~h}$ at $37{ }^{\circ} \mathrm{C}$, the cells were harvested, homogenized in buffer containing $20 \mathrm{mM}$ Tris- $\mathrm{HCl}$ (pH 8.0), $500 \mathrm{mM} \mathrm{NaCl}, 20 \mathrm{mM}$ imidazole, $1 \mathrm{mM}$ PMSF and disrupted by sonication. Cell debris were removed by low-speed centrifugation for $15 \mathrm{~min}$. The supernatant was collected and applied to ultracentrifugation at $150000 \times \mathrm{g}$ for $1 \mathrm{~h}$. The membrane fraction was harvested and suspended in 20 $\mathrm{mM}$ Tris- $\mathrm{HCl}$ (pH 8.0), $500 \mathrm{mM} \mathrm{NaCl}, 20 \mathrm{mM}$ imidazole, $1 \mathrm{mM}$ PMSF. After the addition of Triton X-100 at a final concentration of $1 \%(\mathrm{v} / \mathrm{v})$, the sample was incubated for $10 \mathrm{~h}$ with slow stirring at $4{ }^{\circ} \mathrm{C}$. After another ultracentrifugation step at $150000 \times g$ for $1 \mathrm{~h}$, the supernatant was collected and loaded onto $\mathrm{Ni}^{2+}$-nitrilotriacetate affinity resin (Ni-NTA) and rinsed with buffer containing $20 \mathrm{mM}$ Tris- $\mathrm{HCl}(\mathrm{pH}$ 8.0), $500 \mathrm{mM} \mathrm{NaCl}, 40 \mathrm{mM}$ imidazole and $0.02 \%$ (w/ v) DDM. The protein was eluted from the affinity resin with buffer containing $20 \mathrm{mM}$ Tris- $\mathrm{HCl}, \mathrm{pH} 8.0,500 \mathrm{mM} \mathrm{NaCl}, 300 \mathrm{mM}$ imidazole and $0.02 \%(\mathrm{w} / \mathrm{v}) \mathrm{DDM}$. The protein was concentrated to $5 \mathrm{mg} / \mathrm{ml}$ before further purification by gel filtration (Superdex-200 column, GE Healthcare) in buffer containing $20 \mathrm{mM}$ Tris$\mathrm{HCl}$ (pH 8.0), $500 \mathrm{mM} \mathrm{NaCl}, 2 \mathrm{mM}$ DTT, $0.02 \%$ (w/v) DDM. The peak fractions were collected and concentrated to $3.2 \mathrm{mg} /$ ml. $T t$ NikM2 $2_{1-209}$ (amino acids from 1 to 209) was cloned, overexpressed and purified in the same way. The $T t \mathrm{NikM} 2_{1-209}$ with the divalent cobalt ion was purified by same method but using the CoNTA column instead of the Ni-NTA.

Crystallization, data collection and structural determination Extensive crystallization trials were performed for the purified $T t \mathrm{NikM} 2$ proteins. Crystals were grown at $18{ }^{\circ} \mathrm{C}$ by the hangingdrop vapour diffusion method. After optimization, the full-length $T t \mathrm{NikM} 2$ crystals were grown by mixing $1 \mu \mathrm{l}$ of protein $(3.2 \mathrm{mg} /$ $\mathrm{ml}$ ) with $1 \mu \mathrm{l}$ of reservoir solution (38\% w/v PEG300, $0.05 \mathrm{M}$ glycine, pH9.5, $0.1 \mathrm{M} \mathrm{NaCl}, 0.2 \mu 135 \% \mathrm{w} / \mathrm{v}$ 1,6-hexanediol, $0.2 \mu 16.6 \% \mathrm{w} / \mathrm{v}$ cyclohexylethanoyl-N-hydroxyethylglucamide). $T t$ NikM2 crystals appeared and grew to full size in about one 
week. The $T t \mathrm{NikM} 2_{1-209}$ crystals were grown in the same way except that the concentration of PEG300 in the reservoir solution is $35.5 \% \mathrm{w} / \mathrm{v}$. The $T t \mathrm{NikM} 2_{1-209}$ crystals with cobalt ion were grown in the same way except that the buffer changed to MES with the $\mathrm{pH}$ 6.4. All the crystals were frozen in liquid nitrogen directly to collect the data.

All the crystal data were collected at the Shanghai Synchrotron Radiation Facility (SSRF) BL17U, integrated and scaled using the HKL2000 package [45]. Further processing was carried out using programs from the CCP4 suite [46]. Data collection statistics are summarized in Supplementary information, Table S2 and Table S3. The nickel sites were located using SHELXD [47] from Bijvoet differences in the Ni-SAD data. Heavy atom positions were defined and the phases were calculated with PHASER's SAD (single anomalous diffraction) experimental phasing module [48]. The real-space constraints were applied to the electron density map in DM. The final model rebuilding was performed using COOT [49] and the protein structure was refined with PHENIX [50] using NCS and stereochemistry information as restraints. The structure of full-length $T t \mathrm{NikM} 2$ was solved by molecular replacement method with the $T t \mathrm{NikM} 2_{1-209}$ structure as the search model. All structural figures were generated in PyMOL [51].

\section{XANES data collection}

The concentration of the $T t \mathrm{NikM} 2$ was about $0.5 \mathrm{mM}$. Samples were flash frozen in liquid nitrogen. During the XANES measurement, the sample was maintained at a temperature of $80 \mathrm{~K}$. All measurements of the Ni $K$-edge XANES were carried out on beam line BL14W1 at the Shanghai Synchrotron Radiation Facility (SSRF, China) in the fluorescence yield mode using a double-crystal $\mathrm{Si}(111)$ monochromator and a 32-element array of Ge detectors. A $\mathrm{N}_{2}$-filled ionization chamber was used to measure the incident flux. The storage ring was working at $3.5 \mathrm{GeV}$ and experiments were performed with an electron current $220 \mathrm{~mA}$. Simultaneous energy calibration was performed by measuring the absorption of Ni foil.

The XANES spectrum is highly sensitive to the coordination geometry around the absorbing atom. A shoulder peak appeared a few eV below the main absorption edge in the XANES spectrum which is attributed to the $1 \mathrm{~s}-$ to- $4 \mathrm{p}_{\mathrm{z}}$ electronic transition is a signature of a square-planar complex [36]. The presence or absence of the $1 \mathrm{~s}-$ to- $-4 \mathrm{p}_{\mathrm{z}}$ transition has been used to predict the coordination geometry in several model compounds $[52,53]$.

\section{Construction of RcNik(MN)QO transporter variants and transport assay}

Plasmid $p \operatorname{RcNik}(M N) Q O$ encoding the natural $\operatorname{Nik}(M N)$ fusion, $N i k Q$ and a C-terminally FLAG-tagged $N i k O$ [6] was the basis for all constructions. Individual mutations were introduced by two rounds of PCR. In the first round, amplicons were generated with a mutagenic forward or reverse primer and a corresponding primer directed against the 3 '-end of nikMN or the upstream region in the vector, respectively. Those amplicons were used as primers in a second round of PCR together with the appropriate non-mutagenic primer of the first round. The second-round products were treated with appropriate restriction endonucleases and were used to replace the wild-type fragments. All synthetic DNAs were verified by nucleotide sequencing. Mutant plasmids were introduced into E. coli XL1-Blue and nickel-uptake activity was determined as described [6]. Briefly, cells were grown in lysogeny broth (LB) over night, diluted 1:100 in LB supplemented with ampicillin (100 $\mu \mathrm{g} / \mathrm{ml})$, IPTG $(1 \mathrm{mM})$ and ${ }^{63} \mathrm{NiCl}_{2}(500 \mathrm{nM})$, and incubated under shaking at $37{ }^{\circ} \mathrm{C}$ for seven hours. Cells were harvested, washed twice with Tris-hydrochloride $(50 \mathrm{mM}, \mathrm{pH} 7.5)$ and concentrated. Metal accumulation was quantitated by liquid scintillation counting and is expressed as $\mathrm{pmol} / \mathrm{mg}$ protein.

\section{Quantum chemical analysis}

The theoretical calculations were performed with Gaussian 09 (G09) program [54]. We used the LANL2DZ effective core potentials and corresponding valence basis sets for the metals [55] and 6-311+G* all-electron basis sets for $\mathrm{C}, \mathrm{N}, \mathrm{O}$, and $\mathrm{H}$ [56]. Because of the complexity of $3 \mathrm{~d}$-transition metals, we investigated the binding energies using a variety of approximate exchange-correlation (XC) functionals as benchmark. The XC functionals used include the generalized gradient approximation (GGA) of PerdewBurke-Ernzerhof (PBE) [57], hybrid GGA of PBE0, B3LYP, and $\mathrm{BHaH}$ (Becke's half-and-half hybrid functional), highly nonlocal hybrid meta-GGA functional M06-2X and full Hartre-Fock functional M06-HF [58]. The basic trends of the calculated energies and charges are similar from these $\mathrm{XC}$ functional. Based on the charge and spin-density distribution, the performance of the $\mathrm{BHaH}$ functional is satisfactory and we only present results calculated from this functional. The results are also compared with those calculated using Amsterdam Density Fucntional (ADF) program [59].

The vibrational frequencies were calculated by using harmonic approximation. The zero-point energy (ZPE) is included in the calculated binding energies. The atomic polar tensor (APT) derived charges were calculated to provide the information on charge distribution upon different ligands binding [40]. The energies calculated are listed in Supplementary information, Table S4 and the atomic net charges are listed in Supplementary information, Table S5.

\section{$P D B$ deposition}

The coordinates and diffraction data of $T t \mathrm{NikM} 2_{1-209}$ (with $\left.\mathrm{Co}^{2+}\right), T t \mathrm{NikM}_{1-209}\left(\right.$ with $\mathrm{Ni}^{2+}$ ) and the full-length $T t \mathrm{NikM} 2$ with $\mathrm{Ni}^{2+}$ have been deposited into the Protein Data Bank with accession code 4M5C, 4M5B and 4M58, respectively.

\section{Acknowledgments}

We thank the staffs at the SSRF BL17U, BL14W1 and BSRF $4 \mathrm{~W} 1 \mathrm{~B}$ beamlines for their support in measurements and data reduction. This work was supported by grants from the Ministry of Science and Technology and the National Natural Science Foundation of China to MY (2012CB911101, 2011CB910502, 31030020 and 31170679 ) and by grants from the Deutsche Forschungsgemeinschaft to TE (EI 374/4-1 and EI 374/4-2, within PAK 459). The theoretical work was supported by NKBRSF (2011CB932400) of China. The calculations were done at the Supercomputer Center of the Computer Network Information Center, Chinese Academy of Sciences, and the Shanghai Supercomputing Center.

\section{References}

1 Berntsson RP, ter Beek J, Majsnerowska M, et al. Structural divergence of paralogous S components from ECF-type ABC transporters. Proc Natl Acad Sci USA 2012; 109:13990-13995. 
2 Karpowich NK, Wang DN. Assembly and mechanism of a group II ECF transporter. Proc Natl Acad Sci USA 2013; 110:2534-2539.

$3 \mathrm{Xu} \mathrm{K}$, Zhang M, Zhao Q, et al. Crystal structure of a folate energy-coupling factor transporter from Lactobacillus brevis. Nature 2013; 497:268-271.

4 Wang T, Fu G, Pan X, et al. Structure of a bacterial energycoupling factor transporter. Nature 2013; 497:272-276.

5 Zhang P, Wang J, Shi Y. Structure and mechanism of the S component of a bacterial ECF transporter. Nature 2010; 468:717-720.

6 Rodionov DA, Hebbeln P, Gelfand MS, Eitinger T. Comparative and functional genomic analysis of prokaryotic nickel and cobalt uptake transporters: evidence for a novel group of ATPbinding cassette transporters. J Bacteriol 2006; 188:317-327.

7 Neubauer O, Alfandega A, Schoknecht J, Sternberg U, Pohlmann A, Eitinger T. Two essential arginine residues in the T components of energy-coupling factor transporters. J Bacteriol 2009; 191:6482-6488

8 Erkens GB, Berntsson RP, Fulyani F, et al. The structural basis of modularity in ECF-type ABC transporters. Nat Struct Mol Biol 2011; 18:755-760.

9 Zhang Y, Rodionov DA, Gelfand MS, Gladyshev VN. Comparative genomic analyses of nickel, cobalt and vitamin B12 utilization. BMC Genomics 2009; 10:78.

10 Zhang Y, Gladyshev VN. Comparative genomics of trace elements: emerging dynamic view of trace element utilization and function. Chem Rev 2009; 109:4828-4861.

11 Lunin VV, Dobrovetsky E, Khutoreskaya G, et al. Crystal structure of the CorA $\mathrm{Mg}^{2+}$ transporter. Nature 2006; 440:833837.

12 Higgins KA, Carr CE, Maroney MJ. Specific metal recognition in nickel trafficking. Biochemistry 2012; 51:7816-7832.

13 Sigel A, Sigel H, Sigel RKO. Front Matter. In: Nickel and Its Surprising Impact in Nature, Volume 2. John Wiley \& Sons, Ltd, Chichester, UK, 2007: i-xxvi.

14 Boer JL, Mulrooney SB, Hausinger RP. Nickel-dependent metalloenzymes. Arch Biochem Biophys 2013 Sep 10. doi: 10.1016/j.abb.2013.09.002

15 Rickes EL, Brink NG, Koniuszy FR, Wood TR, Folkers K. Vitamin B12, a Cobalt Complex. Science 1948; 108:134.

16 Bertrand EM, Saito MA, Jeon YJ, Neilan BA. Vitamin B 12 biosynthesis gene diversity in the Ross Sea: the identification of a new group of putative polar $\mathrm{B}_{12}$ biosynthesizers. Environ Microbiol 2011; 13:1285-1298.

17 Randaccio L, Geremia S, Demitri N, Wuerges J. Vitamin B12: unique metalorganic compounds and the most complex vitamins. Molecules 2010; 15:3228-3259.

18 Frenkel EP, Kitchens RL, Johnston JM. The effect of vitamin B12 deprivation on the enzymes of fatty acid synthesis. J Biol Chem 1973; 248:7450-7456.

19 Rachmilewitz M. Neurological manifestations in Vitamin B 12 deficiency. Harefuah 1969; 77:269-271.

20 Eschenmoser A, Wintner CE. Natural product synthesis and vitamin B12. Science 1977; 196:1410-1420.

21 Moore SJ, Lawrence AD, Biedendieck R, et al. Elucidation of the anaerobic pathway for the corrin component of cobalamin (vitamin B12). Proc Natl Acad Sci USA 2013; 110:1490614911.
22 Elofsson A, von Heijne G. Membrane protein structure: prediction versus reality. Annu Rev Biochem 2007; 76:125-140.

23 Heijne G. The distribution of positively charged residues in bacterial inner membrane proteins correlates with the transmembrane topology. EMBO J 1986; 5:3021-3027.

24 Siche S, Neubauer O, Hebbeln P, Eitinger T. A bipartite S unit of an ECF-type cobalt transporter. Res Microbiol 2010; 161:824-829.

25 Hattori M, Tanaka Y, Fukai S, Ishitani R, Nureki O. Crystal structure of the $\mathrm{MgtE} \mathrm{Mg}^{2+}$ transporter. Nature 2007; 448:1072-1075.

26 Eshaghi S, Niegowski D, Kohl A, Martinez Molina D, Lesley SA, Nordlund P. Crystal structure of a divalent metal ion transporter CorA at 2.9 angstrom resolution. Science 2006; 313:354-357.

27 Peters T Jr, Interaction of one mole of copper with the alpha amino group of bovine serum albumin. Biochim Biophys Acta 1960; 39:546-547.

28 Ginotra YP, Ramteke SN, Srikanth R, Kulkarni PP. Mass spectral studies reveal the structure of Abeta1-16- $\mathrm{Cu}^{2+}$ complex resembling ATCUN motif. Inorg Chem 2012; 51:7960-7962.

29 Melino S, Gallo M, Trotta E, Mondello F, Paci M, Petruzzelli R. Metal-binding and nuclease activity of an antimicrobial peptide analogue of the salivary histatin 5. Biochemistry 2006; 45:15373-15383.

30 Gonzalez-Diaz H, Sanchez-Gonzalez A, Gonzalez-Diaz Y. 3DQSAR study for DNA cleavage proteins with a potential antitumor ATCUN-like motif. J Inorg Biochem 2006; 100:12901297.

31 Sankararamakrishnan R, Verma S, Kumar S. ATCUN-like metal-binding motifs in proteins: identification and characterization by crystal structure and sequence analysis. Proteins 2005; 58:211-221.

32 Mal TK, Ikura M, Kay LE. The ATCUN domain as a probe of intermolecular interactions: application to calmodulin-peptide complexes. J Am Chem Soc 2002; 124:14002-14003.

33 Donaldson LW, Skrynnikov NR, Choy WY, et al. Structural characterization of proteins with an attached ATCUN motif by paramagnetic relaxation enhancement NMR spectroscopy. $J$ Am Chem Soc 2001; 123:9843-9847.

34 Gasmi G, Singer A, Forman-Kay J, Sarkar B. NMR structure of neuromedin $\mathrm{C}$, a neurotransmitter with an amino terminal CuII-, NiII-binding (ATCUN) motif. J Pept Res 1997; 49:500509.

35 Harford C, Sarkar B. Neuromedin C binds $\mathrm{Cu}(\mathrm{II})$ and Ni(II) via the ATCUN motif: implications for the CNS and cancer growth. Biochem Biophys Res Commun 1995; 209:877-882.

36 Eidsness MK, Sullivan RJ, Schwartz JR, et al. Structural diversity of F-430 from methanobacterium-thermoauto-trophicum - a nickel X-ray absorption spectroscopic study. J Amer Chem Soc 1986; 108:3120-3121.

37 Johnson ER, Dickson RM, Becke AD. Density functionals and transition-metal atoms. J Chem Phys 2007; 126:184104.

38 Hohenberg P, Kohn W. Inhomogeneous electron gas. Phys Rev 1964; 136:B864-B871.

39 Kohn W, Sham LJ. Self-consistent equations including exchange and correlation effects. Phys Rev 1965; 140:A1133A1138.

40 Cioslowski J. A new population analysis based on atomic polar 
tensors. J Am Chem Soc 1989; 111:8333-8336.

41 Edgar RC. MUSCLE: multiple sequence alignment with high accuracy and high throughput. Nucleic Acids Res 2004; 32:1792-1797.

42 Keane TM, Creevey CJ, Pentony MM, Naughton TJ, McLnerney JO. Assessment of methods for amino acid matrix selection and their use on empirical data shows that ad hoc assumptions for choice of matrix are not justified. BMC Evol Biol 2006; 6:29.

43 Guindon S, Gascuel O. A simple, fast, and accurate algorithm to estimate large phylogenies by maximum likelihood. Syst Biol 2003; 52:696-704.

44 Sole VA, Papillon E, Cotte M, Walter P, Susini J. A multiplatform code for the analysis of energy-dispersive X-ray fluorescence spectra. Spectrochimica Acta Part B: Atomic Spectroscopy 2007; 62:63-68.

45 Otwinowski Z, Minor W. Processing of X-ray diffraction data collected in oscillation mode. Macromolecular Crystallography, Pt A 1997; 276:307-326.

46 Collaborative Computational Project N. The CCP4 suite: programs for protein crystallography. Acta Crystallogr D Biol Crystallogr 1994; 50:760-763.

47 Schneider TR, Sheldrick GM. Substructure solution with SHELXD. Acta Crystallogr D Biol Crystallogr 2002; 58:17721779 .

48 McCoy AJ, Grosse-Kunstleve RW, Adams PD, Winn MD, Storoni LC, Read RJ. Phaser crystallographic software. J Appl Crystallogr 2007; 40:658-674.

49 Emsley P, Cowtan K. Coot: model-building tools for molecular graphics. Acta Crystallogr D Biol Crystallogr 2004; 60:21262132.

50 Adams PD, Grosse-Kunstleve RW, Hung LW, et al. PHENIX: building new software for automated crystallographic structure determination. Acta Crystallogr D Biol Crystallogr 2002; 58:1948-1954.

51 DeLano WL. The PyMOL molecular graphics system. Nature
Publishing Group, a division of Macmillan Publishers. 2002.

52 Chen LX, Jager WJH, Jennings G, Gosztola DJ, Munkholm A, Hessler JP. Capturing a photoexcited molecular structure through time-domain X-ray absorption fine structure. Science 2001; 292:262-264.

53 Colpas GJ, Maroney MJ, Bagyinka C, et al. X-ray spectroscopic studies of nickel-complexes, with application to the structure of nickel sites in hydrogenases. Inorg Chem 1991; 30:920-928.

54 Frisch MJ, Trucks GW, Schlegel HB, et al. Gassing 09, Revision B. 01,. Gassing, Inc., Walling, CT, 2010.

55 Wadt WR, Hay PJ. Abinitio effective core potentials for molecular calculations-potentials for main group elements NA to BI. J Chem Phys 1985; 82:284-298.

56 Krishnan R, Binkley JS, Seeger R, Pople JA. Self-consistent molecular orbital methods. XX. A basis set for correlated wave functions. J Chem Phys 1980; 72:650-654.

57 Perdew JP, Burke K, Ernzerhof M. Generalized gradient approximation made simple. Phys Rev Letters 1996; 77:38653868.

58 Zhao Y, Truhlar DG. The M06 suite of density functionals for main group thermochemistry, thermochemical kinetics, noncovalent interactions, excited states, and transition elements: two new functionals and systematic testing of four M06-class functionals and 12 other functionals. Theor Chem Acc 2008; 120:215-241.

59 te Velde G, Bickelhaupt FM, Baerends EJ, et al. Chemistry with ADF. J Comput Chem 2001; 22:931-967.

(Supplementary information is linked to the online version of the paper on the Cell Research website.)

(c) (1) (s) This work is licensed under the Creative Commons Attribution-NonCommercial-No Derivative Works 3.0 Unported License. To view a copy of this license, visit http:// creativecommons.org/licenses/by-nc-nd/3.0 